LamA is required to modify the mycobacterial cell wall in such a way that, in a single round of replication, half the daughter cells exhibit phenotypic drug tolerance. The mechanisms responsible for this differential drug sensitivity remain to be determined. This genetically encoded capacity to generate heterogeneity at each round of replication could confer a huge advantage for the maintenance of $M$. tuberculosis populations under drug pressure. The authors observed that, when treated with antibiotics, lamA-mutant $M$. tuberculosis cells were killed at a more uniform rate than wild-type M. tuberculosis cells.

Why would a process enabling the generation of heterogeneity in bacterial progeny through the action of LamA be found in Mycobacterium species? We might think of drug tolerance and drug resistance as properties emergent from modern-day antibiotic usage, but antibiotics are natural products that are particularly abundant in soil microbes. Ancestral mycobacterial species inhabiting such environmental niches would have encountered many antibiotic molecules and experienced selective pressures to acquire mechanisms of tolerance and resistance long before bacteria such as $M$. tuberculosis needed to evolve in response to modern chemotherapy.

Understanding the basis of heterogeneity might provide insight into the interplay between host immune-derived pressures and their effect on current antituberculosis treatments. Rego and colleagues' research reveals one mechanism by which heterogeneity might be generated among replicating $M$. tuberculosis, but it is certainly not the only mechanism, and the situation is undoubtedly more complex in an in vivo infection. Host-derived stresses and functional immune responses are known to drive an increase in heterogeneity in M. tuberculosis ${ }^{7}$. Furthermore, M. tuberculosis bacteria in activated host immune cells called phagocytes exhibit enhanced drug tolerance to frontline antituber"The mechanisms culosis compounds responsible compared with bacteria in nonfor this differential drug sensitivity remain to be determined." activated phagocytes $^{8}$. One could speculate that the daughter cells generated by the action of LamA that had low calcein fluorescence could be at an advantage in dealing with both host-cell-induced stress and drug pressure in vivo.

The pathways to drug tolerance may differ in their underlying mechanisms, but the consequences are comparable. Drug tolerance caused by heterogeneity is probably a major driver of the evolution of heritable drug resistance $e^{9}$ because heterogeneity and drug tolerance promote bacterial survival and thereby expand the window of opportunity for the acquisition of mutations that reduce antibiotic efficacy.
Although the prospects for developing successful treatments for tuberculosis remain daunting, understanding the biological basis of drug tolerance might open new avenues through which antibiotics could be used in combination with compounds that block the capacity of $M$. tuberculosis to promote heterogeneity or mobilize its drug-tolerance programs ${ }^{10}$. Drug-discovery approaches need to move beyond the landscape of target-based screening and embrace a discovery process that incorporates and tests the complex biology of bacterial heterogeneity and drug tolerance that occurs in vivo.

David G. Russell is in the Department of Microbiology and Immunology, Cornell University, Ithaca, New York 14853, USA. e-mail:dgr8@cornell.edu

1. World Health Organization. Global Tuberculosis Report 2016 (World Health Organization, 2016).

2. Rego, E. H., Audette, R. E. \& Rubin, E. J. Nature 546 , 153-157 (2017).

3. Kester, J. C. \& Fortune, S. M. Crit. Rev. Biochem. Mol. Biol. 49, 91-101 (2014)

4. Verstraeten, N., Knapen, W., Fauvart, M. \& Michiels, J. Methods Mol. Biol. 1333, 3-13 (2016).

5. Aldridge, B. B. et al. Science 335, 100-104 (2012)

6. Maglica, Ž., Özdemir, E. \& McKinney, J. D. MBio 6, e02236-14 (2015).

7. Manina, G., Dhar, N. \& McKinney, J. D. Cell Host Microbe 17, 32-46 (2015).

8. Liu, Y. et al. J. Exp. Med. 213, 809-825 (2016)

9. Levin-Reisman, I. et al. Science $\mathbf{3 5 5}, \mathbf{8 2 6}-830$ (2017).

10.Zheng, H. et al. Nature Chem. Biol. 13, 218-225 (2017).

\title{
Premature lambs grown in a bag
}

\section{A bag that mimics the environment of the womb can support lambs born extremely prematurely for four weeks. This development points to ways to improve the prospects of extremely premature babies.}

\section{CLAIRE T. ROBERTS}

$\mathrm{E}$ ach year, about 15 million babies worldwide are born prematurely ${ }^{1}$, of whom about $9 \%$ are extremely premature, born after fewer than 30 weeks of gestation ${ }^{2}$. Research to develop an artificial womb in which to house extremely premature babies began in the 1960s, but no such device has yet been implemented ${ }^{3}$. Difficulties include contamination of the fluid in which babies must be submerged, which can cause infection, and pressure imbalances in the fetal blood vessels and heart caused by the device that provides oxygen, which can lead to heart failure. Writing in Nature Communications,
Partridge et al. ${ }^{4}$ report a step in the right direction - the development of a fluid-filled 'Biobag' into which they delivered lambs born at a stage equivalent to extremely premature human infants.

The Biobag is a sealable polythene bag in which synthetic amniotic fluid containing antibiotics is circulated in a sterile environment, preventing infections (see Fig. 1 of the paper ${ }^{4}$ ). The authors placed eight extremely premature lambs in Biobags immediately after birth, and connected blood vessels from the animals' umbilical cords to a specialized pumpless oxygenator device. Blood flow around the device is driven by the lamb's heart rather than by a pump, mimicking the rate of blood flow between the fetus and placenta in utero, and so preventing pressure imbalances in the fetal heart and blood vessels. In addition, immediate incubation in a closed fluid environment prevents a cascade of changes that normally occur when the newborn takes its first breath, filling the lungs with air. These changes lead to re-routing of the circulation such that oxygenation occurs through the lungs, rather than from the placenta through the umbilical cord. Thus, the Biobag permits retention of the fetal circulation - no mean feat.

Following birth, newborns no longer receive a continuous nutrient supply from the placenta, so the researchers provided nutrients intravenously. Some lambs also received insulin, which promotes fetal growth ${ }^{5}$ and regulates blood glucose levels. These animals grew at a similar rate to controls that developed in utero. Extremely premature human infants typically grow more poorly than they would in utero, so this is an impressive result.

Lambs were grown in Biobags for up to four weeks, and it is possible that longer incubation times would be feasible. Although such survival is an aim of neonatal intensive care units, survival without disability is the ultimate goal. Encouragingly, incubated lambs showed near-normal heart function. Oxygen delivery to the fetus and levels of the oxygen-carrying 

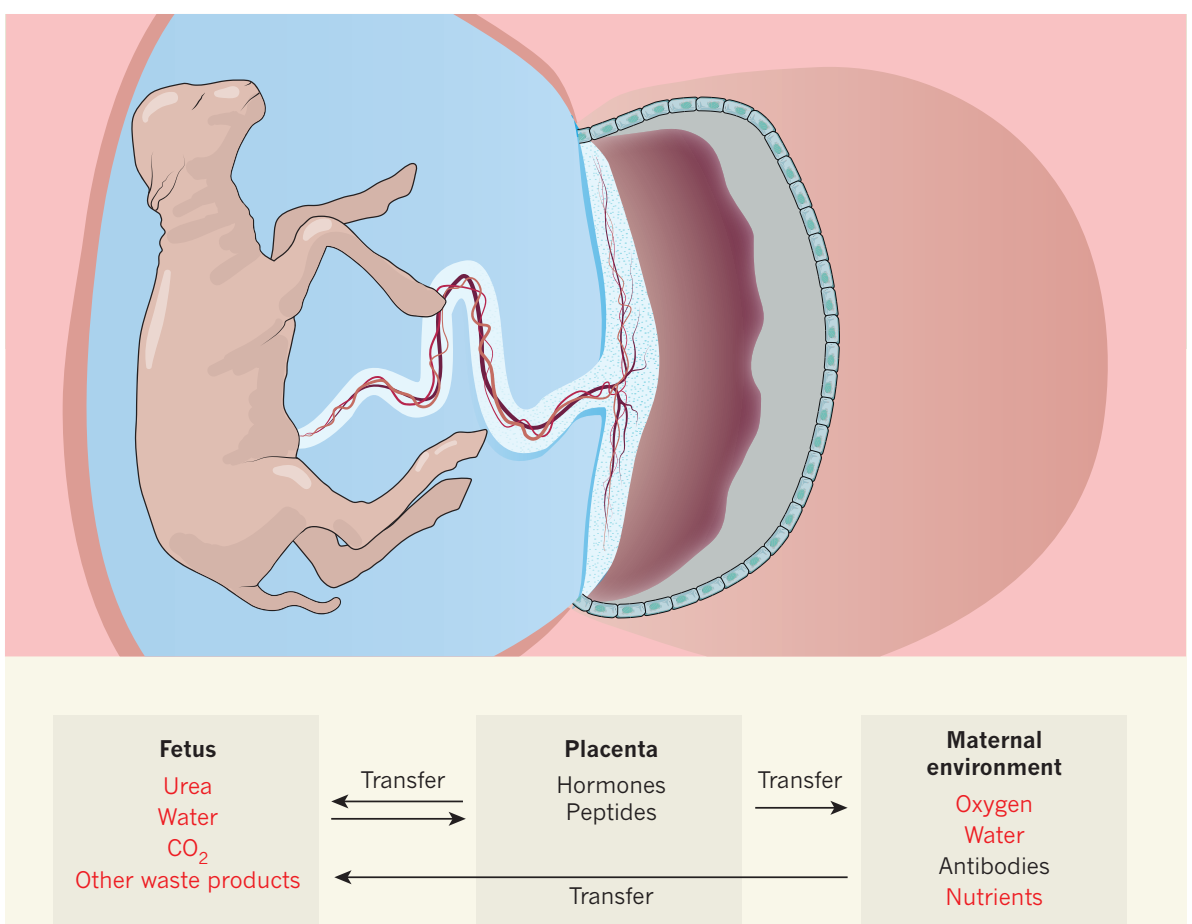

Figure 1 | Mother-placenta-fetus crosstalk during mammalian development. During mammalian pregnancies, blood vessels in the umbilical cord are the conduit for complex crosstalk between the mother, placenta and fetus. Oxygen, nutrients and maternal antibodies and hormones are transferred to the developing fetus to promote healthy growth, and waste products, including urea and carbon dioxide, are removed. Placental hormones and growth factors promote fetal growth and proper organ development, and orchestrate maternal adaptations to pregnancy. Partridge et al. ${ }^{4}$ designed an artificial womb called a Biobag (not shown) in which they could grow lambs born extremely prematurely. Their set-up could mimic some aspects of mother-placenta-fetus crosstalk (those in red), but development is required to more fully mimic the interactions seen in utero.

protein haemoglobin in red blood cells were normal at the beginning of incubation, but gradually diminished over time. However, this defect could be ameliorated by administering the hormone erythropoietin, which promotes the production of red blood cells.

A newborn's lungs must produce a detergent-like substance called surfactant to reduce surface tension and prevent collapse during exhalation, which leads to respiratory distress syndrome. In extremely premature babies, medical intervention is typically required to ensure that the underdeveloped lungs secrete surfactant. Partridge and colleagues' immediate incubation allowed the lungs to continue to develop as if still in utero. Post-mortem analysis revealed that the lungs continued normal structural development and began to produce surfactant. Moreover, lambs were readily transferred to a ventilator after delivery from the Biobag, as would be needed for extremely premature human infants, who may still require assistance to breathe after Biobag incubation. Normal lung development is a major achievement, because respiratory distress syndrome is life-threatening for premature infants and can result in lifelong chronic lung disease.

Premature babies are also at risk of cognitive defects ${ }^{6}$. Partridge et al. provide some

reassurance that the growth and differentiation of key brain areas seemed to be normal. The authors found no evidence of brain injury, which is often seen in premature infants. Moreover, brain structure and function were normal in a single lamb assessed at six months old. However, these results should be interpreted with caution. The brains of lambs develop differently from those of humans, and lambs

\section{"Many hurdles must be overcome before Biobags can be consideredfor humanuse."} cannot be used to assess bleeding in the brain - a common problem in human premature infants that, when severe, can lead to neurological disabilities.

Partridge and colleagues' results are promising, and suggest that the Biobag could soon be used as a tool in basic research for determining causal mechanisms of premature birth and for testing potential therapies for babies in utero. This could be done in lambs and potentially in other species. However, many hurdles must be overcome before Biobags can be considered for human use.

For instance, women at risk of premature labour are prescribed glucocorticoid hormones, which help the baby's lungs and other organs to mature in preparation for life outside the womb. The lambs were not exposed to prenatal glucocorticoids, and inducing early lung maturation might prevent successful incubation in a Biobag. Given that glucocorticoid therapy is well established and highly successful at preventing respiratory distress syndrome ${ }^{7}$, the idea of not using this treatment would be difficult to sell to doctors.

Another caveat is that many extremely premature births are attributable to infections of the uterus, which are often undiagnosed ${ }^{1}$. These infections can have long-term consequences for infants. Biobag protocols would need to be tailored to match the needs of infected infants or of those born extremely prematurely for other reasons - for example, if the baby is induced because the mother has severely high blood pressure, a condition called pre-eclampsia.

Although the Biobag can deliver oxygen and nutrients and remove waste, in its current form it does not replace other placental functions (Fig. 1). These include the transfer of myriad placental hormones, growth factors and, importantly, maternal antibodies that provide protection from infections ${ }^{8}$. Future Biobag prototypes should incorporate these functions.

A final consideration is whether parents would be happy to use Biobags. Current treatments for extremely premature infants involve placing babies in an incubator on a ventilator, which can be upsetting for highly stressed parents. One wonders how parents would react to the prospect of placing their baby in a water-filled plastic bag.

Partridge et al. suggest that the Biobag would be best suited to babies born from 23 to 25 weeks' gestation, who are at high risk of dying or surviving with a disability. The Biobag is clearly innovative - if successfully refined, it could show promise for use in large, wellresourced neonatal intensive care units.

Claire T. Roberts is at Adelaide Medical School, Robinson Research Institute, University of Adelaide, Adelaide, South Australia 5005, Australia. e-mail: claire.roberts@adelaide.edu.au

1. Romero, R., Dey, S. K. \& Fisher, S. J. Science 345, 760-765 (2014).

2. Verburg, P. E. et al. PLOS ONE 11, e0158807 (2016).

3. Schoberer, M. et al. Artif. Organs 36, 512-516 (2012).

4. Partridge, E. A. et al. Nature Commun. 8, 15112 (2017).

5. Sferruzzi-Perri, A. N., Vaughan, O. R., Forhead, A. J. \& Fowden, A. L. Curr. Opin. Clin. Nutr. Metab. Care 16, 298-309 (2013).

6. March of Dimes, PMNCH, Save the Children \& WHO. Born Too Soon: The Global Action Report on Preterm Birth (eds Howson, C. P., Kinney, M. V. \& Lawn, J. E.) (WHO, 2012).

7. Roberts, D., Brown, J., Medley, N. \& Dalziel, S. R. Cochrane Database Syst. Rev. 3, CDd004454 (2017).

8. Gude, N. M., Roberts, C. T., Kalionis, B. \& King, R. G. Thromb. Res. 114, 397-407 (2004). 